PROCEEDINGS OF THE

AMERICAN MATHEMATICAL SOCIETY

Volume 137, Number 8, August 2009, Pages 2575-2580

S 0002-9939(09)09826-8

Article electronically published on February 6, 2009

\title{
FREYD'S GENERATING HYPOTHESIS WITH ALMOST SPLIT SEQUENCES
}

\author{
JON F. CARLSON, SUNIL K. CHEBOLU, AND JÁN MINÁČ \\ (Communicated by Birge Huisgen-Zimmermann)
}

\begin{abstract}
Freyd's generating hypothesis for the stable module category of a non-trivial finite group $G$ is the statement that a map between finitely generated $k G$-modules that belongs to the thick subcategory generated by the field $k$ factors through a projective module if the induced map on Tate cohomology is trivial. In this paper we show that Freyd's generating hypothesis fails for $k G$ when the Sylow $p$-subgroup of $G$ has order at least 4 using almost split sequences. By combining this with our earlier work, we obtain a complete answer to Freyd's generating hypothesis for the stable module category of a finite group. We also derive some consequences of the generating hypothesis.
\end{abstract}

\section{INTRODUCTION}

The second and third authors have studied the generating hypothesis (GH) and related questions for the stable module category of a finite group in a series of papers 5, 2, 6, 7] with Benson and Christensen, and have obtained many partial results. In this paper we give a complete solution to the generating hypothesis for the stable module category of a finite group. We begin by recalling the statement of the GH. Let $G$ be a finite group and let $k$ be a field of characteristic $p$. We will work in the stable module category $\operatorname{stmod}(k G)$ of $k G$. Recall that this is the tensor triangulated category obtained from the category of finitely generated left $k G$-modules by factoring out the projective modules. In the stable module category, $\underline{\operatorname{Hom}}_{k G}(A, B)$ stands for the space of maps between modules $A$ and $B$, and $\Omega$ will denote the translation functor. Loosely speaking, the generating hypothesis claims that if a module $L$ generates a subcategory, then the functor $\underline{\operatorname{Hom}}_{k G}\left(\Omega^{*} L,-\right)$ detects trivial maps (maps that factor through a projective module) in the subcategory. We make this precise in the case that is of interest to us, namely the subcategory generated by the trivial representation $k$. Let thick ${ }_{G}(k)$ denote the thick subcategory generated by $k$, that is, the smallest full subcategory of $\operatorname{stmod}(k G)$ that contains the trivial module $k$ and is closed under exact triangles and direct summands. The Generating Hypothesis $(\mathrm{GH})$ for a group ring $k G$ is the statement that the Tate

Received by the editors June 12, 2008, and, in revised form, October 21, 2008.

2000 Mathematics Subject Classification. Primary 20C20, 20J06; Secondary 55P42.

Key words and phrases. Tate cohomology, generating hypothesis, stable module category, ghost map, almost split sequence.

The first author is partially supported by a grant from the NSF.

The third author is supported by the NSERC.

(C)2009 American Mathematical Society Reverts to public domain 28 years from publication 
cohomology functor $\hat{\mathrm{H}}^{*}(G,-) \cong \underline{\operatorname{Hom}}_{k G}\left(\Omega^{*} k,-\right)$

$$
\begin{aligned}
\operatorname{thick}_{G}(k) & \longrightarrow \hat{\mathrm{H}}^{*}(G, k) \text {-modules } \\
M & \mapsto \hat{\mathrm{H}}^{*}(G, M)
\end{aligned}
$$

detects trivial maps in $\operatorname{thick}_{G}(k)$, i.e., that the Tate cohomology functor $\hat{\mathrm{H}}^{*}(G,-)$ on $\operatorname{thick}_{G}(k)$ is faithful. Note that when $G$ is a $p$-group, then thick $(k)$ is the entire stable module category $\operatorname{stmod}(k G)$.

It is shown in 2 that the GH holds for $k P$ when $P$ is a $p$-group if and only if $P$ is $C_{2}$ or $C_{3}$. It is natural then to conjecture that for an arbitrary finite group $G$ the GH holds for $k G$ if and only if the Sylow $p$-subgroup of $G$ is either $C_{2}$ or $C_{3}$. In [6] this conjecture has been proved using block theory for groups which have periodic cohomology. In this paper, we show that the GH fails for groups with non-periodic cohomology. In fact, we show that the GH fails whenever the Sylow $p$-subgroup of $G$ has order at least 4 . Thus we have a complete solution to Freyd's generating hypothesis in the stable module category:

Theorem 1.1. Let $G$ be a finite group and let $k$ be a field of characteristic $p$ that divides the order of $G$. Then the $G H$ holds for $k G$ if and only if a Sylow p-subgroup of $G$ is isomorphic to either $C_{2}$ or $C_{3}$.

Our main tool in showing the failure of the GH is an almost split sequence. In contrast with our earlier counting techniques $[\underline{6}$ which only show the existence of a counter-example to the GH, our current treatment with almost split sequences has the advantage that it produces an explicit and simple counter-example whenever the GH fails.

For the interested reader we mention that there has been great interest in the generating hypothesis in other triangulated categories including the stable homotopy category of spectra [8] where it originated, but also in the derived categories of rings [10, 9].

Throughout the paper $G$ will denote a finite group, and $k$ will be a field of characteristic $p$ which divides the order of $G$. We use the standard facts about the stable module category of $k G$, which can be found in 3 .

The first author thanks the Alexander von Humboldt Foundation for support and the RWTH in Aachen for their hospitality while part of this paper was written.

\section{The GENERATING HYPOTHESIS}

A ghost in thick $(k)$ is a map between $k G$-modules in thick $(k)$ that induces the trivial map in Tate cohomology. Our goal is to show that there are non-trivial ghosts in thick $(k)$ whenever the Sylow $p$-subgroup of $G$ has order at least 4 . Our main tool in showing the existence of these non-trivial ghosts is an almost split sequence (a.k.a. Auslander-Reiten sequence) which we now define.

A short exact sequence

$$
\epsilon: \quad 0 \longrightarrow A \longrightarrow B \longrightarrow C \longrightarrow 0
$$

of finitely generated $k G$-modules is an almost split sequence if $A$ and $C$ are indecomposable $k G$-modules, and $\epsilon$ is a non-split sequence with the property that every map $M \rightarrow C$ which is not a split epimorphism factors through the middle term $B$ 1]. It is a theorem of Auslander and Reiten that given any finitely generated indecomposable non-projective $k G$-module $C$, there exists a unique (up to isomorphism 
of short exact sequences) almost split sequence terminating in $C$. Moreover, the first term $A$ of the almost split sequence ending in $C$ is shown to be isomorphic to $\Omega^{2} C$.

The theorem that we are now interested in is the following.

Theorem 2.1. Let $M$ and $N$ be two non-projective indecomposable modules in $\operatorname{stmod}(k G)$ such that $N \approx \Omega^{i}(M)$ for any $i$. Then there exists a non-trivial map $\phi: N \longrightarrow \Omega N$ in $\operatorname{stmod}(k G)$ such that the induced map

$$
\phi_{*}: \widehat{\operatorname{Ext}}_{k G}^{*}(M, N) \longrightarrow \widehat{\operatorname{Ext}}_{k G}^{*}(M, \Omega N)
$$

is the zero map.

Proof. Consider the almost split sequence

$$
0 \longrightarrow \Omega^{2} N \longrightarrow B \longrightarrow N \longrightarrow 0
$$

ending in $N$. This short exact sequence represents a distinguished triangle

$$
\Omega^{2} N \longrightarrow B \longrightarrow N \stackrel{\phi}{\longrightarrow} \Omega N
$$

in the stable category. We will show that the map $\phi: N \longrightarrow \Omega N$ has the desired properties. Almost split sequences are, by definition, non-split short exact sequences, and therefore the boundary map $\phi$ in the above triangle must be a non-trivial map in the stable category.

The next thing to be shown is that the map $\phi: N \longrightarrow \Omega N$ induces the zero map on the functors $\underline{\operatorname{Hom}}_{k G}\left(\Omega^{i} M,-\right) \cong \widehat{\operatorname{Ext}}^{i}(M,-)$ for all $i$. To this end, consider any map $f: \Omega^{i} M \longrightarrow N$. We have to show that the composite

$$
\Omega^{i} M \stackrel{f}{\longrightarrow} N \stackrel{\phi}{\longrightarrow} \Omega N
$$

is trivial in the stable category. Consider the following diagram:

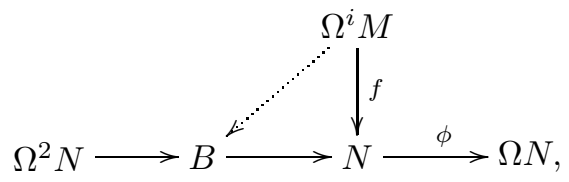

where the bottom row is our distinguished triangle. The map $f: \Omega^{i} M \longrightarrow N$ cannot be a split epimorphism by the given hypothesis; therefore by the defining property of an almost split sequence, the map $f$ factors through the middle term $B$ as shown in the above diagram. Since the composite of any two successive maps in a distinguished triangle is zero, the composite $\phi \circ f$ is also zero by commutativity. So we are done.

Corollary 2.2. If there is an indecomposable non-projective module $N$ in thick $k_{G}(k)$ which is not isomorphic to $\Omega^{i} k$ for any $i$, then the $G H$ fails for $k G$.

Proof. We apply the previous theorem with $M=k$. Since $\widehat{\operatorname{Ext}}_{k G}^{*}(k, N) \cong$ $\hat{\mathrm{H}}^{*}(G, N)$, the existence of an indecomposable non-projective module $N$ in thick $G(k)$ with the property that $N \approx \Omega^{n} k$ for any $n$ implies (by the above theorem) the existence of a non-trivial map $\phi: N \longrightarrow \Omega N$ in $\operatorname{thick}_{G}(k)$ such that the induced map of $\hat{\mathrm{H}}^{*}(G, k)$-modules, $\hat{\mathrm{H}}^{*}(G, N) \longrightarrow \hat{\mathrm{H}}^{*}(G, N)$, is the zero map. In other words $\phi: N \longrightarrow \Omega N$ is a non-trivial ghost in thick $(k)$. Therefore the GH fails for $k G$. 
It is not hard to produce modules that satisfy the conditions laid out in the above corollary. Specifically we have the following.

Theorem 2.3. Suppose that the Sylow p-subgroup $P$ of $G$ has order at least 4. Then the GH fails for $k G$.

Proof. We divide the proof into two cases. First suppose that $\mathrm{H}^{*}(G, k)$ is periodic, implying that $P$ is either cyclic or quaternion. By Tate duality, there must exist a non-zero element $\zeta \in \hat{\mathrm{H}}^{n}(G, k)$ for some odd integer $n$. Then we have an exact sequence

$$
\mathcal{E}_{\zeta}: \quad 0 \longrightarrow L_{\zeta} \longrightarrow \Omega^{n} k \stackrel{\zeta}{\longrightarrow} k \longrightarrow 0,
$$

where $\zeta$ in the sequence is a cocycle representing the cohomology element $\zeta$, and $L_{\zeta}$ is the kernel of $\zeta$. In the case that $P$ is cyclic, $\Omega^{n} k$ when restricted to $P$ is a direct sum of a projective module and a uniserial module of dimension $|P|-1$. Because the cohomology element $\zeta$ is not zero, its restriction to $P$ is not zero and the restriction of $L_{\zeta}$ is the direct sum of a projective module and a uniserial module of dimension $|P|-2$. Consequently, $L_{\zeta}$ must be indecomposable and, moreover, because its dimension is not 1 or -1 modulo $|P|, L_{\zeta}$ is not isomorphic to $\Omega^{n} k$ for any $n$. So by Corollary 2.2, the GH fails.

If $P$ is a quaternion group, then again by Tate duality, we can assume that $n$ is positive and congruent to -1 modulo 4 . When we restrict $L_{\zeta}$ to $P$ we get the direct sum of a projective module and a copy of $\operatorname{Rad}\left(\Omega^{-1} k\right)$, which is easily seen to be indecomposable. So by the same argument as before, the GH fails.

Now suppose that the cohomology ring $\mathrm{H}^{*}(G, k)$ is not periodic. This means that the maximal ideal spectrum $V_{G}(k)$ of the cohomology ring $\mathrm{H}^{*}(G, k)$ has Krull dimension at least two. This time we choose $\zeta \in \mathrm{H}^{n}(G, k)$ with the property that $n>0$ and $\zeta$ is not nilpotent. Then we construct the sequence $\mathcal{E}_{\zeta}$ and the module $L=L_{\zeta}$ exactly as in the periodic case. The support variety of the module $L$ is equal to $V_{G}(L)=V_{G}(\zeta)$, the collection of maximal ideals that contain the element $\zeta$ [4. (Note here that we do not need to assume that the field $k$ is algebraically closed, though the proof of the statement about the variety of $L_{\zeta}$ requires extending the scalars to the algebraically closed case.) Because $\zeta$ is not nilpotent, $V_{G}(L)$ is a proper subvariety of $V_{G}(k)$ and the same statement will hold for any direct summand of $L$. Hence, if $U$ is any indecomposable direct summand of $L$, we must have that $U \nRightarrow \Omega^{n}(k)$ for all $n$, simply because the support varieties are different. Consequently by Corollary 2.2 the GH cannot hold for $k G$.

\section{Consequences of the GH}

We now derive some consequences of the GH. First of all, we would like to point out that our main result implies that the GH for $k G$ depends only on the characteristic of the field $k$. In other words, if $k_{1}$ and $k_{2}$ are two fields of characteristic $p$ which divide the order of $G$, then the GH holds for $k_{1} G$ if and only if it holds for $k_{2} G$. It is not clear how one would prove this fact directly.

The dual generating hypothesis is also a natural problem to ask. That is, instead of using the (covariant) Tate cohomology functor $\underline{\operatorname{Hom}}_{k G}\left(\Omega^{*} k,-\right)$, we can use the (contravariant) dual Tate cohomology functor $\underline{\operatorname{Hom}}_{k G}\left(-, \Omega^{*} k\right)$ and ask if this contravariant functor detects trivial maps in thick ${ }_{G}(k)$. The duality functor $M \mapsto M^{*}$ sets a tensor triangulated equivalence between $\operatorname{thick}_{G}(k)$ and its opposite category 
thick $_{G}(k)^{\text {opp }}$. In particular, the GH holds for $k G$ if and only if the dual GH holds for $k G$. Combining this with our previous results we have:

Theorem 3.1. The following assertions are equivalent:

(1) The Sylow p-subgroup of $G$ is either $C_{2}$ or $C_{3}$.

(2) The functor $\underline{\operatorname{Hom}}_{k G}\left(\Omega^{*} k,-\right)$ is faithful on $\operatorname{thick}_{G}(k)$.

(3) The functor $\underline{\operatorname{Hom}}_{k G}\left(-, \Omega^{*} k\right)$ is faithful on $\operatorname{thick}_{G}(k)$.

(4) $\operatorname{thick}_{G}(k)$ consists of finite direct sums of suspensions of $k$.

Proof. (1) $\Longrightarrow$ (2) is shown in [6, (2) $\Longrightarrow$ (4) is shown in Corollary [2.2, (4) $\Longrightarrow(2)$ is trivial, and $(2) \Longrightarrow(1)$ is shown in Theorem 2.3 . Finally the equivalence of statements (2) and (3) is shown in the paragraph preceding this theorem.

Our final result is motivated by a result of Peter Freyd [8] which states that if the stable homotopy functor on the category of finite spectra is faithful, then it is also full. We now prove the analogue of this statement for the stable module category. This generalizes Theorem 3.3 of [2, where we established the same result for $p$-groups.

Theorem 3.2. If the $G H$ holds for $k G$, then the Tate cohomology functor from thick $_{G}(k)$ to the category of modules over $\hat{\mathrm{H}}^{*}(G, k)$ is also full.

Proof. If the GH holds for $k G$, then from the equivalence $(2 \Longleftrightarrow 4)$ of Theorem 3.1 we know that thick ${ }_{G}(k)$ is made up of finite direct sums of suspensions of $k$. In particular, for each $M$ in thick $G(k), \hat{\mathrm{H}}^{*}(G, M)$ is a free $\hat{\mathrm{H}}^{*}(G, k)$-module of finite rank. It follows that the induced map

$$
\underline{\operatorname{Hom}}_{k G}(M, X) \longrightarrow \operatorname{Hom}_{\hat{\mathrm{H}}^{*}(G, k)}\left(\hat{\mathrm{H}}^{*}(G, M), \hat{\mathrm{H}}^{*}(G, X)\right)
$$

is an isomorphism for all $k G$-modules $X$. Since $M$ was an arbitrary $k G$-module in thick ${ }_{G}(k)$, we have shown that the functor $\hat{\mathrm{H}}^{*}(G,-)$ is full, as desired.

The class of groups for which the GH has an affirmative answer (namely, groups whose Sylow $p$-subgroup is $C_{2}$ or $C_{3}$ ), although small, has some interesting candidates. This includes interesting simple groups. For example, it has the smallest simple group $A_{5}$ of order 60 in characteristic 3. Another interesting example in characteristic 3 is the smallest Janko group $J_{1}$ which has order 175560 . In fact, $J_{1}$ is the only sporadic simple group for which the GH holds.

\section{Symmetric Algebras}

We end the paper by pointing out that much of the work here applies for any finite dimensional symmetric algebra. Note that if $A$ is such an algebra, we have almost split sequences ending in any finitely generated indecomposable non-projective $A$-module $N$ :

$$
0 \longrightarrow \Omega^{2} N \longrightarrow E \longrightarrow N \longrightarrow 0 .
$$

Therefore our proofs generalize immediately to give the following result.

Theorem 4.1. Let $A$ be a finite dimensional symmetric $k$-algebra. Fix a finitely generated non-projective indecomposable A-module $L$. Then the following are equivalent. 
(1) The functor $\underline{\operatorname{Hom}}_{A}\left(\Omega^{*} L,-\right)$ is faithful on thick $(L)$, the thick subcategory generated by $L$ in $\operatorname{stmod}(A)$.

(2) thick $(L)$ consists of modules isomorphic to finite direct sums of suspensions of $L$.

We sketch a proof and leave the details to the reader.

Proof. Clearly (2) implies (1). For the other direction, suppose that (2) fails. Then we have a an indecomposable non-projective object $N$ in thick $(L)$ such that $N \nsucceq$ $\Omega^{i} L$ for any $i$. Then the map $N \longrightarrow \Omega N$ corresponding to the almost split sequence ending in $N$ is shown (exactly as before) to be a non-trivial map that is invisible to the functor $\underline{\operatorname{Hom}}_{A}\left(\Omega^{*} L,-\right)$. This shows that (1) fails. So we are done.

\section{REFERENCES}

[1] M. Auslander, I. Reiten, and S. Smalø. Representation Theory of Artin Algebras. Cambridge Studies in Advanced Mathematics 36, 1995. MR.1314422 (96c:16015)

[2] David Benson, Sunil K. Chebolu, J. Daniel Christensen, and Ján Mináč. The generating hypothesis for the stable module category of a p-group. Journal of Algebra, 310(1):428-433, 2007. MR2307802 (2007k:16011)

[3] Jon F. Carlson. Modules and group algebras. Lectures in Mathematics ETH Zürich. Birkhäuser Verlag, Basel, 1996. Notes by Ruedi Suter. MR.1393196 (97c:20013)

[4] J. F. Carlson. The variety of an indecomposable module is connected. Invent. Math., 77(1984), 291-299. MR752822 (86b:20009)

[5] Sunil K. Chebolu, J. Daniel Christensen, and Ján Mináč. Groups which do not admit ghosts. Proc. Amer. Math. Soc., 136(4):1171-1179, 2008. MR2367091(2008k:20017)

[6] Sunil K. Chebolu, J. Daniel Christensen, and Ján Mináč. Freyd's generating hypothesis for groups with periodic cohomology. Preprint, 2007. arXiv:0710.3356

[7] Sunil K. Chebolu, J. Daniel Christensen, and Ján Mináč. Ghosts in modular representation theory. Advances in Mathematics, 217:2782-2799, 2008. MR2397466 (2008m:20018)

[8] Peter Freyd. Stable homotopy. In Proc. Conf. Categorical Algebra (La Jolla, Calif., 1965), pages 121-172. Springer, New York, 1966. MR0211399 (35:2280)

[9] Mark Hovey, Keir Lockridge, and Gena Puninski. The generating hypothesis in the derived category of a ring. Mathematische Zeitschrift, 256(4):789-800, 2007. MR2308891 (2008b:18017)

[10] Keir Lockridge. The generating hypothesis in the derived category of $R$-modules. Journal of Pure and Applied Algebra, 208(2):485-495, 2007. MR2277690 (2007i:55009)

Department of Mathematics, University of Georgia, Athens, Georgia 30602

E-mail address: jfc@math.uga.edu

Department of Mathematics, Illinois State University, Normal, Illinois 61790

E-mail address: schebol@ilstu.edu

Department of Mathematics, University of Western Ontario, London, On N6A 5B7, CANADA

E-mail address: minac@uwo.ca 